\title{
Abstracts of Award-Winning Posters, 13th Annual Health Sciences Poster Conference, Faculty of Medicine, Health Sciences Centre, Kuwait University, Kuwait, April 22-24, 2008
}

\section{Dr. Nael Al-Naqeeb Award for Best Undergraduate Research}

\section{Knowledge, Beliefs and Attitude towards Consanguinity and Premarital Screening for Hereditary Diseases among Kuwaiti University Students}

H. Al-Baaly, A. Al-Khateeb, L. Al-Mutairi, E. Al-Zayer

Department of Community Medicine and Behavioural Sciences, Faculty of Medicine, Kuwait University, Kuwait

Introduction: The aim of this study is to assess the three components of knowledge and attitude (knowledge of hereditary diseases, knowledge of premarital screening, and attitude towards consanguinity) in Kuwaiti university students that could potentially modulate their attitude towards premarital screening. Subjects and Methods: A cross-sectional survey with a sample size of 592 Kuwaiti university students, using a gender-stratified multistage cluster sample was carried out during March-April, 2007. Data were collected through a self-administered questionnaire. Multiple regression analyses were used to evaluate the independent associations in the study. Results: The overall rate of consanguinity between students' parents was $41.9 \%$. Family history of a hereditary disease was present in $42.3 \%$ of the participants. $58.8 \%$ of participants were not in favour of consanguineous marriages. Customs and traditions were perceived to be the main factors behind the preference of consanguineous marriage (95.3\%). 89\% of participants were aware of the existence of premarital screening services. The majority of the participants agreed that premarital screening should be mandatory and that it does not conflict with religious teaching. Multivariate logistic regression analysis iden- tified the following factors to be associated with the favourable attitude towards premarital screening: good knowledge of hereditary diseases $(\mathrm{AOR}=1.8,95 \% \mathrm{CI} 1.2-2.7)$, good knowledge of premarital screening $(\mathrm{AOR}=1.7,95 \% \mathrm{CI} 1.2-2.5)$, and non-favourable attitude towards consanguinity $(\mathrm{AOR}=1.6,95 \% \mathrm{CI} 1.1-$ 2.5). Conclusions: Premarital screening was perceived as an acceptable measure in the control of hereditary diseases not conflicting with religious, social, or cultural factors. This study found that good knowledge of hereditary diseases, good knowledge of premarital screening, and non-favourable attitude towards consanguinity were independently related to a favourable attitude towards premarital screening.

\section{Best Postgraduate Awards}

\section{Basic Sciences (MSc)}

\author{
Microarray Profiling of siRNA-Knockout of RKIP in \\ Primary HEK293s Cell Line \\ Z. Taqi ${ }^{\text {a }}$, W. Al-Ali ${ }^{\text {b }, ~ S . ~ J a c o b ~}{ }^{\text {b }}$, F. Al-Mulla ${ }^{\text {b }}$ \\ a MSc student in Program of Molecular Biology, \\ College of Graduate Studies, and b Department of Pathology, \\ Faculty of Medicine, Kuwait University, Kuwait
}

Introduction: The Raf kinase inhibitor protein (RKIP) is a member of a conserved group of proteins called PEBP. On the molecular level, RKIP is an inhibitor of the Raf/MEK/ERK signaling pathway. Initially, RKIP was identified as a metastasis suppressor gene in human prostate cancer. Our laboratory demon-

\section{KARGER}

Fax +4161306 1234 E-Mail karger@karger.ch www.karger.com (c) 2008 S. Karger AG, Basel

1011-7571/08/0176-0507\$24.50/0

Accessible online at: www.karger.com/mpp 
strated for the first time an association between loss of RKIP expression and reduced survival in early-stage colorectal cancer and metastasis in breast cancer. Although the molecular mechanism by which RKIP inhibits the Raf signaling pathway has been partially delineated, little is known about the biological relevance and consequences of silencing RKIP in primary cells. Our research addresses a significant niche in the RKIP literature and presents novel data on the mechanism of RKIP functions. Materials and Methods: RKIP was down-regulated by transfecting normal HEK293 cells with pcDNA6. 2 plasmids coding for RKIPmiRNA. The level of RKIP protein in standard HEK293 and miRNA-knockout cell line was detected by Western immunoblotting. Both the growth rate and migration assays were performed. In addition, HEK293 cell RNA was used in microarray profiling. U133 Plus 2 Affymetrix GeneChip was utilized to determine the expression profile of the genes in relation to the expression or down-regulation of RKIP. Finally, real-time PCR was used to further confirm and quantify the detected gene expression changes. Results: The reduction of RKIP expression in miRNA-knockout cells was verified by Western blotting and realtime PCR. In growth curve assay, miRNA-knockout cells grew faster. Moreover, miRNA-RKIP-silenced HEK cells had a higher migration index than control cells. To understand the molecular defects responsible for these phenotypic changes, we compared the expression profiling of control versus RKIP-silenced cells, in triplicate, using Affymetrix high-density U133 Plus 2 GeneChip. Our data show significant differences in expression profiling between the two cell lines $(\mathrm{p}<0.0000001)$, mainly in relation to genes that control metabolism, growth, differentiation, apoptosis and oxidative stress. These novel data sets are discussed in relation to cancer growth, invasion and metastasis. Conclusions: We present novel data pertaining to the function of RKIP protein and the catastrophic consequences of its down-regulation on the behavior of primary cells.

\section{Basic Sciences (PhD)}

\section{Heterogeneous Adenovirus Serotypes Cause Conjunctivitis in Kuwait}

A.I. Al-Rifaiy ${ }^{\text {a }, ~ J . ~ A l-M e r j a n ~}{ }^{\text {b }}$, G. Szucs ${ }^{\text {a }}$, W.S. Al-Nakib ${ }^{\text {a }}$, A. Pacsa ${ }^{\text {a }}$

avirology Unit, Department of Microbiology, Faculty of Medicine, Kuwait University, and ${ }^{\mathrm{b}} \mathrm{Al}-\mathrm{Bahar}$ Ophthalmology

Center, $\mathrm{MOH}$, Kuwait

Introduction: Different adenovirus (AdV) serotypes cause variable forms of conjunctivitis. Fifty-two human AdV were identified based on neutralization assay. A neutralization epitope has been located on the hypervariable region-7 (HVR-7) of the hexon protein. Hence, HVR-7 has been used as a target for typing of AdV by sequencing. Despite the significance of AdV eye infections, they have never been investigated in Kuwait. Here we used PCR sequencing of HVR-7 to type AdV from conjunctivitis patients, and subsequently identify the amino acids of the neutralization epitope within the HVR-7. Materials and Methods: A total of 163 eye swabs were collected from 131 patients with conjunctivitis. Samples were screened for AdV by PCR using primers specific for the hexon gene conserved region. DNA form AdV-positive samples was amplified by PCR using HVR-7-specific primers. The HVR-7 was sequenced and analyzed by Beckman Coulter CEQ 8000 . The amino acid epitope sequence was deduced from nucleotide sequences within the HVR-7, and both nucleotide and amino acid sequences were compared to sequences available at the GenBank. Results: Sixty-eight samples (41.7\%) were positive for AdV DNA by PCR. Out of 52 randomly selected samples investigated by sequencing, serotypes $7,3,11,8,37,4$ and 5 were identified in $21,11,10,4,3,2$ and 1 sample, respectively. The HVR-7 of local isolates varied in serotypes 7,11 , and 3 , while it showed $100 \%$ homology among serotypes 4, 37 and 8. A case of AdV transmission was confirmed based on identical sequences of isolates from first-degree relatives. HVR-7 neutralizing epitope was identical in local isolates of serotypes $3,4,11,8$ and 37 , while it showed distinctive conservative and nonconservative variations among serotype 7 isolates. Conclusions: This study has identified, for the first time in Kuwait, a heterogeneous population of human AdV serotypes causing eye disease. Serotype 7 was the most dominant $\mathrm{AdV}$ causing conjunctivitis in Kuwait.

\section{Resident}

\section{Scintigraphic Patterns of Poliomyelitis on Bone Scan}

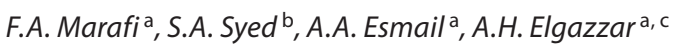

Departments of a Nuclear Medicine and ${ }^{\text {bS Surgery, }}$

Mubarak Alkabeer Hospital, Ministry of Health, and

'Department of Nuclear Medicine, Faculty of Medicine,

Kuwait University, Kuwait

Introduction: Postpoliomyelitis syndrome causes variable musculoskeletal manifestations including pain, muscle weakness and fatigue. These manifestations are commonly secondary to overuse and misuse of muscles and joints and could follow falls. Bone scan can be useful in determining the underlying cause and follow-up. The objective of this study was to identify the scintigraphic patterns of poliomyelitis on bone scan. Materials and Methods: Bone scans of 7 adult patients ( 6 females and 1 male), aged 35-53 years, known to have paralytic poliomyelitis were studied. 6 patients had unilateral while 1 had bilateral disease. All patients had three-phase bone scan and 5 had SPECT study as well. Studies were reviewed by 2 qualified nuclear medicine physicians and findings were recorded and analyzed. Results: Several patterns were consistently identified: (a) significantly decreased uptake of blood pool activity on the affected lower limb of all patients; (b) deformed ipsilateral hemipelvis with reduced uptake on the affected side in all patients, which was less prominent in the patient with bilateral disease; (c) stress changes with increased uptake in the bones of the contralateral lower extremity; (d) degenerative changes of multiple joints (shoulder, knee, hip, ankle and spine). Significant scoliosis was only noted in the patient with bilateral disease. Conclusions: Poliomyelitis-related scintigraphic patterns on bone scan were identified. Awareness of these characteristic scintigraphic findings is important for proper interpretation, thereby avoiding misinterpretation. 


\section{Basic and Applied Sciences Award}

\section{1 \\ Microarray-Based Transcriptome Analysis Reveals \\ Global Gene Upregulation Associated with Kidney \\ Damage in DOCA-Salt-Induced Hypertensive Rats \\ Occurs via Signaling Cascades Involving Epidermal Growth Factor Receptor and RasGTPase}

I.F. Benter ${ }^{\text {a }}$,H. Canatan ${ }^{\text {a }}$, M.H.M. Yousif a , J.T. Anim ${ }^{\text {b }}$, S. Akhtar ${ }^{\text {a }}$

${ }^{a}$ Department of Pharmacology and Toxicology and

${ }^{b}$ Department of Pathology, Faculty of Medicine,

Kuwait University, Kuwait

Introduction: End-organ damage including renal dysfunction is a natural consequence of hypertension. In an attempt to understand the molecular mechanisms leading to renal dysfunction, we analyzed global gene expression changes in the kidneys of rats with deoxycorticosterone acetate (DOCA)-salt-induced hypertension with and without treatment with AG1478, an inhibitor of epidermal growth factor receptor tyrosine kinase (EGFR), or with FPTIII, an inhibitor of RasGTPase. Materials and Methods: Microarray-based global gene expression analysis was performed in triplicate for each rat kidney taken from the following four animal groups: (1) normal (nonhypertensive) Wistar rats, (2) DOCA-hypertensive (DH) rats, (3) DH rats treated with AG1478 and (4) DH rats treated with FPTIII. Amersham Codelink arrays housing 10,000 rat genes were hybridized with RNA taken from rat kidney samples as per manufacturers' instructions. Microarrays were scanned using an Affymetrix Scanner and data analyzed using Imagene and Genowiz (Ocimum Biosolutions) software. Results: Up-regulation of 2,398 genes and down-regulation of only 10 genes by more than 2 -fold were observed in hypertensive rat kidneys compared to nondiseased controls. Interestingly, treatment of animals with AG1478 or FPTIII prevented up-regulation of all genes associated with hypertension in the rat kidney, implying that EGFR and RasGTPase signaling are important mediators of renal damage in hypertension. Histopathological examination confirmed that renal damage induced by hypertension was prevented following treatment with either AG1478 or FPTIII. Conclusions: EGFR and RasGTPase-mediated signaling appear to be important cascades leading to multiple gene changes that ultimately cause renal dysfunction and end-organ damage in hypertension. Therapeutic strategies inhibiting either of these signaling molecules may represent novel paradigms for the treatment of renal disease associated with hypertension.

\section{Characterization and Immunogenicity of RD1 Encoded Antigens of Mycobacterium tuberculosis}

S.N.M. Hanif, R. Al-Attiyah, A.S. Mustafa

Department of Microbiology, Faculty of Medicine, Kuwait University, Kuwait

Introduction: There is an urgent need to identify and characterize new Mycobacterium tuberculosis antigens useful for specific diagnosis and developing safer vaccines against tuberculosis (TB). This study aimed to investigate whether the major/moderate antigens of $M$. tuberculosis encoded by RD1 genes, i.e. ORF3, ORF5, ORF6, ORF7 and RD903, could be useful in the specific diagnosis and/or in developing new vaccines against TB. Methods: DNA corresponding to all genes were cloned in plasmid vectors and expressed in Escherichia coli. The expressed proteins were purified and used to generate antigen-specific antibodies in rabbits and reverse transcriptase-PCR was used to detect gene expression in mycobacteria at protein and mRNA levels, respectively. The diagnostic potential of the proteins was investigated by studying delayed type hypersensitivity (DTH) responses in guinea pigs infected with $M$. tuberculosis, BCG, Mycobacterium avium and Mycobacterium vaccae. Constructed DNA vaccines (pUMVC6 and pUMVC7) based on these genes were evaluated for their effectiveness in mice by studying Th1 and Th2 cytokine responses. Results: RT-PCR and antigen-specific antibodies showed that mRNAs and proteins corresponding to all ORFs were expressed in M. tuberculosis, however only RD903 was expressed in BCG and none were expressed in M. avium and M. vaccae. In guinea pigs, DTH responses to all proteins were observed in animals immunized with $M$. tuberculosis, to RD903 only in BCG-immunized animals and to none of the proteins in animals immunized with M. avium and M. vaccae. Immunization of mice with DNA vaccine constructs of all ORFs sensitized spleen cells to proliferate in response to in vitro stimulation with the purified proteins and cocktails of overlapping synthetic peptides. Spleen cells secreted the protective Th1 cytokine IFN- $\gamma$ but did not secrete nonprotective/pathological Th2 cytokines IL-5/IL-10 in response to the immunizing antigens. Conclusions: This study confirms in vitro and in vivo expression of RD1 ORF3, ORF5, ORF6, and ORF7, and RD903 in M. tuberculosis and suggests their potential as reagents for specific diagnosis and/or developing safer vaccines against TB. 


\section{Clinical Sciences Awards}

1

\section{Scimitar Syndrome: The Impact of Interruption of Anomalous Systemic Arterial Supply on Further Management of Symptomatic Infants}

B. Uthaman ${ }^{\mathrm{a}, \mathrm{b}}$, L. Abushaban ${ }^{\mathrm{a}, \mathrm{b}}$, M. Al-Qbandi ${ }^{\mathrm{b}}$, A. Al-Hay ${ }^{\mathrm{b}}$, J. Rathinasamy ${ }^{\mathrm{b}}$, J. Selvan ${ }^{\mathrm{b}}$

${ }^{a}$ Departments of Medicine and Pediatrics, Faculty of Medicine, Kuwait University, and ${ }^{\mathrm{b}}$ Department of Pediatric and Congenital Cardiology, Chest Hospital, Kuwait

Introduction: There are conflicting sporadic reports in the literature on the impact of interruption of anomalous systemic arterial supply (ASAS) in the management of Scimitar syndrome (SS) presenting during infancy. No systematic study has been undertaken so far on this issue. Methods: Out of 25 children diagnosed to have SS during the past 25.5 years, 17 symptomatic infants had ASAS. After interrupting ASAS (coil embolization 15, surgical ligation 2), they were prospectively evaluated to decide on further management strategies. Results: All 17 infants had sizable ASAS and 9 had variable Scimitar vein (SV) stenosis. Sixteen (94\%) had pulmonary hypertension and significant left-to-right shunt. After intervention, there was variable reduction of shunt in 15 and pulmonary artery pressure in 16 cases. All showed varying clinical improvement. One died of septicemia shortly afterwards. Definitive surgery was deferred for optimal results in 7 children for a mean period of 8 months (range 1 month to 3 years). The remaining 9 children did not require definitive surgery. One among them had closure of the stenosed partial SV by Amplatzer duct occluder ('physiologic correction'). Three children on shortterm and 6 children on long-term follow-up (mean 5.2 years; range 3.3-10.3 years) are doing well. Conclusions: Interruption of ASAS helps to avoid or defer definitive surgery for SS during infancy. Therefore, we recommend coil embolization of ASAS as initial palliation, and long-term medical follow-up to assess need for further intervention.

2

\section{Effect of Obesity on Outcome of Antioxidant Therapy in Men with Asthenozoospermia}

\author{
A.E.Omu ${ }^{\text {a }, M . K . ~ A l-A z e m i ~}{ }^{\text {a }}$, E.O. Kehinde ${ }^{\text {b }}$, J.T.Anim ${ }^{\text {c, }}$ \\ M.A. Oriowo ${ }^{\text {, }}$ T.C. Mathew ${ }^{\mathrm{e}}$ \\ Departments of abstetrics and Gynaecology,

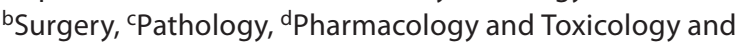 \\ eAnatomy (Electron Microscopy Unit), Faculty of Medicine, \\ Health Sciences Centre, Kuwait University, Kuwait
}

Introduction: Obesity has been associated with male infertility, but the mechanisms are still not fully understood. The objective of the study was to determine the effects of obesity on the outcome of antioxidant therapy in the treatment of asthenozoospermic men and the effect on oxidative stress and apoptosis and sperm fertilizing capacity in male subfertility. Methods: Fortyone men with asthenozoospermia, i.e. $<20$ million $/ \mathrm{ml}$ and $>40 \%$ being immotile, were randomized into four weight-related therapy groups using the body mass index $(\mathrm{BMI}) \leq 18,18.1-25.9,26-$ 29.9 , $\geq 30 \mathrm{~kg} / \mathrm{m}^{2}$. Methodology: Semen analysis according to WHO guidelines, malondialdehyde (MDA), tumor necrosis factor- $\alpha$ (TNF- $\alpha$ ), total antioxidant capacity (TAC), superoxide dismutase (SOD) and glutathione peroxidase were determined in the semen and serum. Sperm chromatin integrity was measured using Sperm Chromatin Structural Assay to assess acid-induced denaturation of sperm DNA and acridine orange staining and evaluated by light microscopy. The second portion was fixed in glutaraldehyde and evaluated by electron microscopy. Results: Obesity was significantly associated with oxidative stress, compared to normozoospermia, with higher seminal levels of MDA and TNF- $\alpha$, and low TAC, SOD and glutathione peroxidase. Markers of oxidative stress, apoptosis and DNA fragmentation index were significantly more common in obese men than in those with normal BMI. Although antioxidant therapy was generally associated with improved sperm parameters, there was significantly less improvement in obese men in terms of reduction of oxidative stress, apoptosis and sperm DNA fragmentation. Conclusions: Obesity was significantly associated with seminal oxidative stress, apoptosis and sperm DNA fragmentation and their reduction by antioxidant therapy was significantly less in obese men than in men with normal body weight.

\section{3}

\section{Establishment of High Throughput Screening for MLH1 and MSH2 Gene Mutations for Detection of HNPCC}

M.J.Marafie a, S.A.Al-Awadi ${ }^{a}$, F. Al-Mosawi ${ }^{\text {a }}$, W. Al-Ali ${ }^{\text {b, }}$ A. Behbehani ${ }^{\text {b }}$,F. Al-Mulla ${ }^{\text {b }}$

${ }^{a}$ Kuwait Medical Genetic Centre, Maternity Hospital, Ministry of Health, and 'bepartment of Pathology, Faculty of Medicine, Kuwait University, Kuwait

Introduction: Colorectal cancer (CRC) is the third most common cancer in the Western world. In Kuwait the incidence of CRC is 8 in 100,000 individuals per year. CRC can either be sporadic or inherited. MLH1 and MSH2 genes are involved in the mismatch repair mechanism. Mutations in these genes have been linked to CRC development. This study aims to establish a high throughput algorithm to detect mutations in these genes via studying and coupling protein expression to targeted DNA sequencing. This approach provides more accurate risk calculation for members with a family history of CRC. Methods: Immunohistochemistry for MLH1 and MSH2 proteins was performed on clinical samples from probands with positive family history of HNPCC. PCR and capillary sequencing methods were optimized for all exons of the MLH1 and MSH2 genes. DNA was collected from members of a family with a history of CRC. Sequencing was carried out on DNA samples of family members and unrelated 
controls. Results: We identified the defective protein in probands prior to sequencing of the target gene, which reduced the mutation detection time by $4-8$ weeks. We tested this workflow algorithm on two families with HNPCC and found a significant reduction in time, effort and money. This approach enabled the confirmation of a heterozygous mutation $(226 \mathrm{C}>\mathrm{T})$ in $\mathrm{MSH} 2$ exon 2 that was found among affected members of a family with history of CRC. Conclusions: This is the first high throughput sequence analysis optimized for MLH1 and MSH2 genes in $\mathrm{Ku}-$ wait coupled to an intelligent protein-based algorithm. This approach serves in enhancing patients' care and counseling of family members on their risk of developing cancer.

\section{Case Report Award}

\section{Intracranial Calcification in Central Diabetes Insipidus: Case Report}

\section{S.R. Al-Kanderi, T. Pandey}

Department of Clinical Imaging, Al-Razi Hospital, $\mathrm{MOH}$, Kuwait

Background: Diabetes insipidus (DI) generally presents with polyuria and polydipsia. However, these are uncommon in infants and presentation is usually nonspecific with features of dehydration, failure to thrive and developmental delay. The diagnosis is, therefore, often delayed till hypernatremia is noted. Imaging in these cases has very little role to play, but rarely characteristic intracranial calcification (ICC) develops which may help in making a diagnosis. The calcifications are seen symmetrically in the basal ganglia and the gray-white matter junctional regions of the frontal, parietal, temporal and occipital lobes. ICC is a known but ex- tremely rare complication of DI. Till now, only 16 cases have been reported and all such cases had the peripheral (nephrogenic) type of diabetes insipidus (NDI). Here we report, for the first time in the literature, a case of ICC complicating central diabetes insipidus (CDI). We also report a case of NDI, comparing the pattern of intracranial calcification in these 2 cases as they appear on CT scans. Case Summary: Patient 1: A 4-year-old boy was admitted in a state of shock due to dehydration. There was history of polyuria and polydipsia of 1-month duration. Investigations revealed the presence of severe hypernatremia and metabolic acidosis. Cerebrospinal fluid analysis and culture were normal. Developmental history revealed evidence of delayed milestones. CT scan of the brain showed multiple symmetric heavily calcified deposits in the basal ganglia and subcortical white matter of the frontal and parieto-occipital lobes. Based on the biochemical and electrolyte changes, a diagnosis of CDI was suspected and confirmed by a positive desmopressin test. The patient was also thoroughly investigated for other possible metabolic or genetic disease but no positive results were obtained. Other causes of intracranial calcifications were excluded by appropriate tests. Patient 2: A 2-month-old child with fever was admitted with the clinical suspicion of acute meningitis for which a CT scan of the brain was obtained and revealed normal findings. His CSF analysis was also normal. His developmental milestones were normal initially but started to lag after 10 months of age. He was readmitted at the age of 15 months with fever, dehydration and breathing difficulty. Investigations revealed the presence of hypernatremia, metabolic acidosis and renal failure. The urine was persistently hypotonic and unresponsive to vasopressin administration. The diagnosis of primary NDI was made after excluding secondary causes. A brain CT obtained at this time showed symmetrical calcifications similar to those seen in case 1 . Conclusion: We found that both the central and peripheral types of DI could give rise to ICC essentially indistinguishable from each other and that the spectrum of imaging findings correlates well with the clinical abnormalities in these conditions. It is important also to maintain a high index of suspicion of DI whenever ICC is seen in a child in the presence of urinary symptoms and developmental delay. 